\title{
Endoscopic Resection of Symptomatic Degenerative Repetitive Stress Related Fracture of the Tip of Superior Articular Process. An Unusual Cause of Severe Back Pain: Report of 2 Cases and Technical Note
}

Hyeun Sung Kim ${ }^{1}$, Pang Hung $\mathrm{Wu}^{1,2}$, Harshavardhan Dilip Raorane ${ }^{1}$, Dong Hwa Heo', Yeon Jin Lee, Kyung-Hoon Yang ${ }^{1}$, Il-Tae Jang ${ }^{1}$

${ }^{1}$ Department of Neurosurgery, Spine Surgery, Nanoori Gangnam Hospital, Seoul, Korea, ${ }^{2}$ National University Health Systems, Jurong Health Campus, Singapore

Corresponding Author:

Hyeun-Sung Kim, MD, PhD

Department of Neurosurgery,

Nanoori Hospital Gangnam, 731,

Eonju-ro, Gangnam-gu, Seoul 06048, Korea

Tel: $+82-2-6003-9767$

Fax: +82-2-3445-9755

E-mail: neurospinekim@gmail.com

"Dr. Hyeun-Sung Kim and Dr. Pang

Hung Wu contributed equally to this work as first authors.

Received: November 27, 2019

Revised: March 26, 2020

Accepted: April 22, 2020
Neglected stress related fracture of superior articular process fracture leading to disability is not commonly reported. Intravertebral foraminal ligament attachment to superior articular process is well described but its repetitive stress related avulsion injury was not reported. The presence of abundant sensory and nociceptive nerve endings around the facet joint can cause significant symptoms in this uncommon condition. We hereby present 2 cases of superior articular process tip fracture. First case is a 53 years old business who presented with intractable chronic back and right gluteal pain for many years worsened with golf games with the diagnosis of nonunion of right $L 2$ superior articular facet and second case is a 74 years old lady who had intractable left buttock and left calf radicular pain with neglected left L5 superior articular facet tip fracture after vertebroplasty treatment on $\mathrm{L} 4$ compression fracture; both underwent uniportal endoscopic contralateral approach for decompression and resection of the Superior Articular Process, SAP tip fragment with its attached foraminal ligament with good symptoms relief on one year follow up. Consideration can be made for endoscopic decompression SAP tip fracture in comparison to open decompression or fusion surgery in these symptomatic patients.

Key Words: Spine fracture, Endoscopic spine surgery, Sports injury, Superior articular process, Foraminal ligament, Repetitive stress fracture, Lumbar endoscopic unilateral laminotomy for bilateral decompression

\section{INTRODUCTION}

Each pair of facet joints in lumbar spine has significant biomechanical contribution to stability and range of motion ${ }^{5}$. Disruption of the integrity of facet joint can lead to significant morbidity to the patient ${ }^{15}$. Fractures of lumbar spine posterior elements usually involved the pedicle or pars articularis. Spondylolysis association with athletes is a popular hypothesis with good literature support ${ }^{4}$. However scarce literature described superior articular process fracture nonunion in athletes ${ }^{7,16)}$ and non-athletes ${ }^{14)}$. Intravertebral foraminal ligament attachment to superior articular process (SAP) is well described in literature, but there is no reported cases of its avulsion injury in our knowledge $^{2,20)}$. Treatment options in this uncommonly described condition range from decompression to fusion. The literature on endoscopic management of this condition is not available to the best of our knowledge. We report 2 cases of endoscopic decompression treatment in this uncommon condition.

\section{CASE REPORT}

\section{Patient 1}

53 years old business man and an avid amateur golf player, 
presented with predominantly back dominant pain with radiation to the right gluteal region for many years. There was no radicular leg pain. Patient was unable to recall any major injury preceding to his pain. His pain was aggravated by playing golf and in flexion and extension movement of his lumbar spine. His medical history was otherwise unremarkable. His physical examination showed tenderness over the region of L1 Vertebra, no motor or sensory deficit, his straight leg raise was limited to 30 degree due to hamstring and back pain. Results of lumbar spine radiography was showing a speck of bony fragment on the superior articular process of right L2 vertebra in lateral and right oblique view on the background of lumbar spine spondylosis changes (Fig. 1). No spinal instability was demonstrated in flexion and extension view. Computer Tomography (CT) scan showed fleck of bone with rounded edges at the right L2 SAP tip with a gap from the main part of SAP, a feature suggestive of chronic
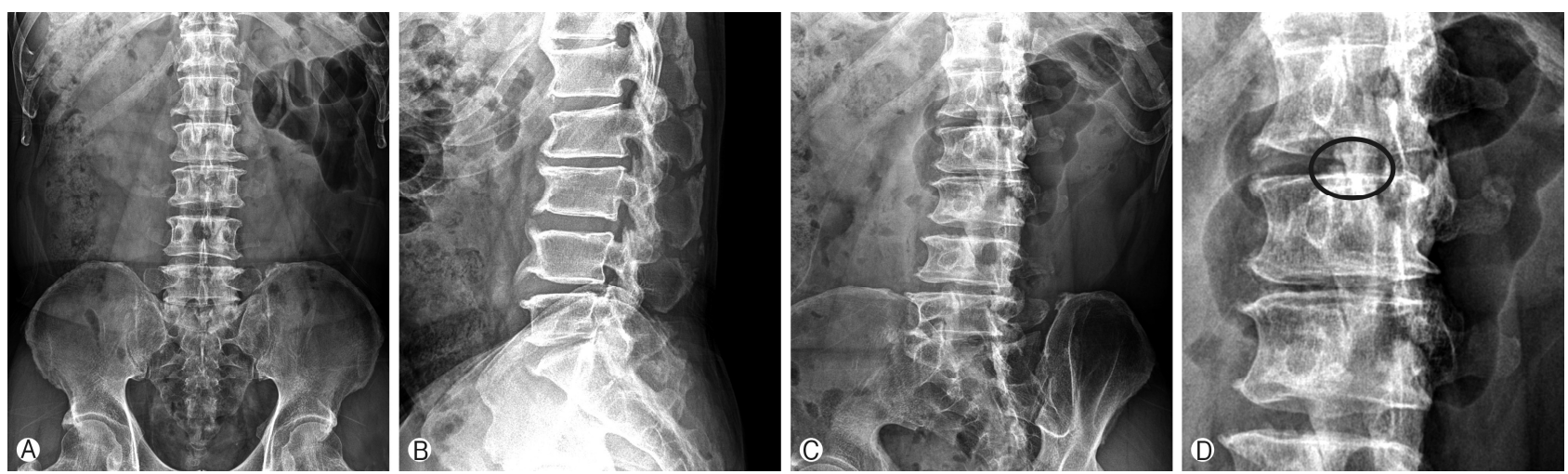

Fig. 1. Case 1 Pre-operative lumbar XR images. Background spondylosis changes in lumbar spine with subtle sign of a fleck of bone with rounded edges at the tip of SAP in lateral view and oblique view which is seen better when magnified (Fig. 1(D), circled in black)
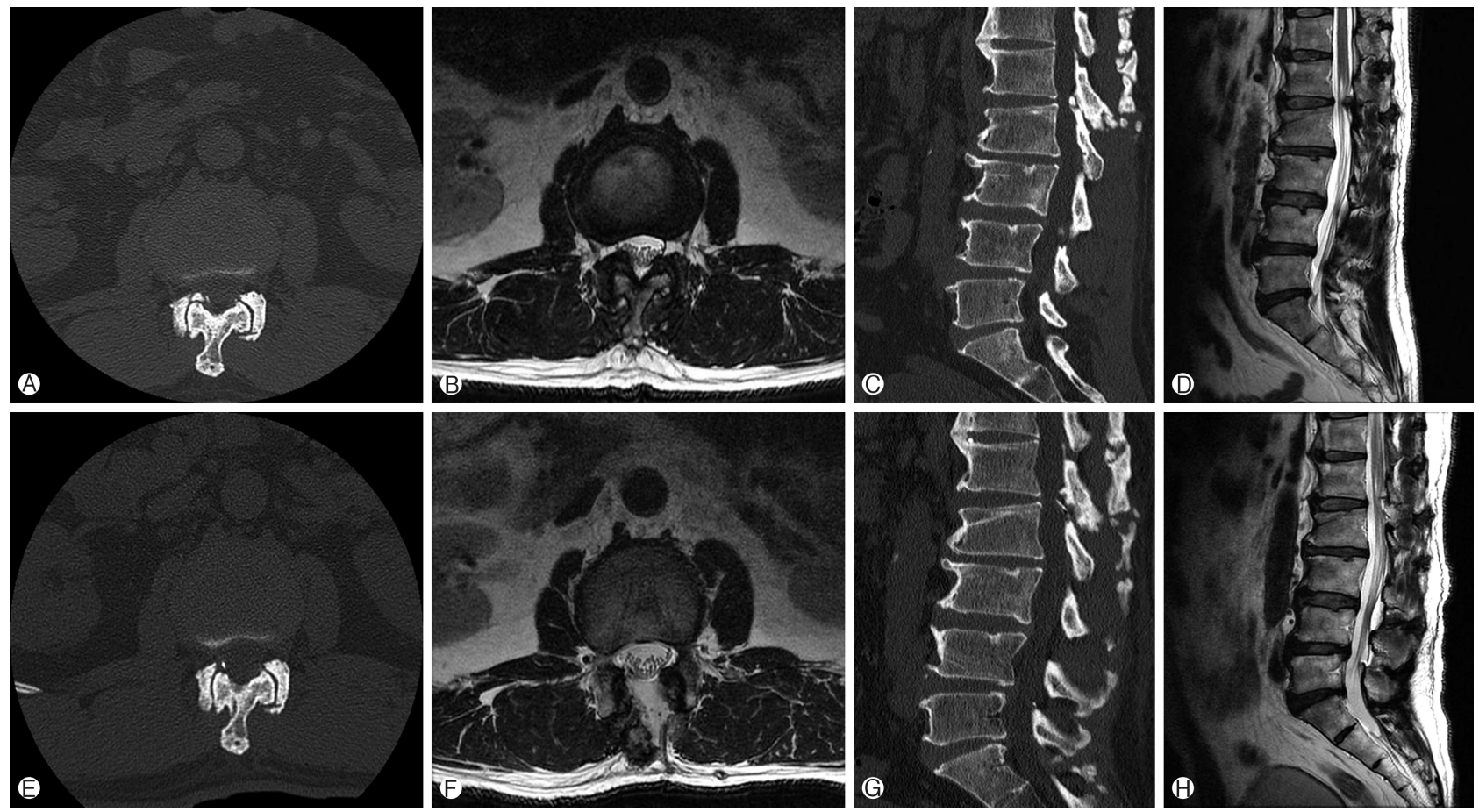

Fig. 2. Case 1, CT and MRI axial and sagittal cuts. A L2 right SAP fleck of bone with rounded edges at the tip of SAP with a gap from the main part of SAP, a feature suggestive of non-union of SAP tip. (B) Corresponding MRI cut showing the non-united fragment abutment of the right traversing nerve root $L 2$ nerve root. (C) and (D) Sagittal cut of CT and corresponding MRI cut showing the fleck of non-united right L2 SAP abutting the traversing L2 nerve root. (E)- $(\mathbb{D}$ showing corresponding cuts with decompression of $\mathrm{L} 1 / 2$ segment and resection of the non-united right SAP bone fragment. 

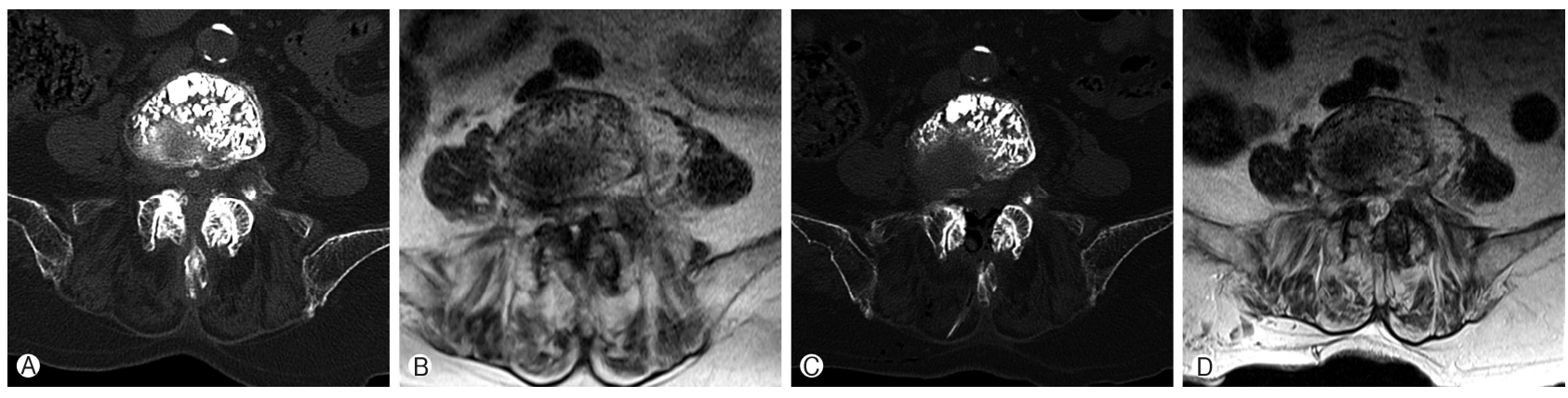

Fig. 3. Case $2 C T$ and MRI axial cuts. (A)-B CT and MRI axial cut showing L4/5 Spinal stenosis with left SAP tip fracture and compression of the left L5 traversing nerve root in the lateral recess. (C)-(D) Corresponding CT and MRI cut post-operative endoscopic stenotic lumbar decompression and contralateral approach resection of left L5 SAP tip fragment showing satisfactory decompression of the L4/5 spinal stenosis.

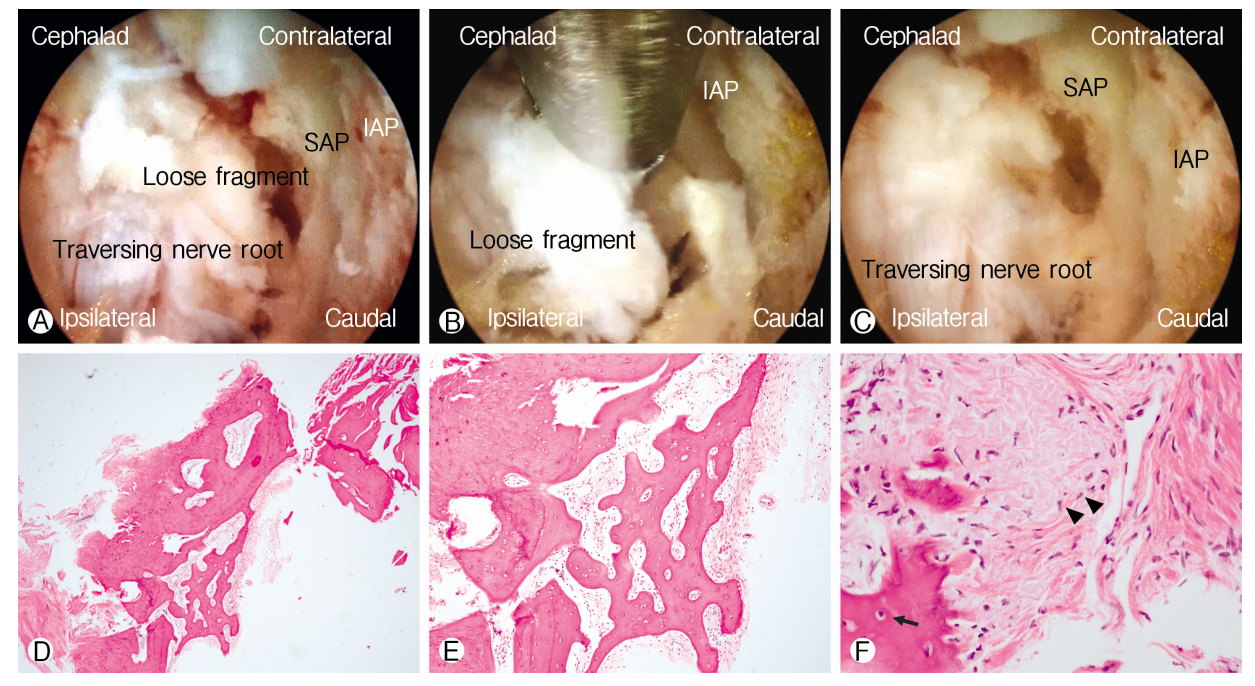

Fig. 4. (A-C) Endoscopic images of case 2. (A) Left L5 loose fragment derived from the non-united left L5 SAP tip fracture is seen after contralateral ligamentum flavum resection. (B) loose fragment is retrieved with endoscopic kerisson rongeur. (C) Decompressed segment showing traversing nerve root relationship with overlying adhesive scar tissue derived from the loose left L5 SAP tip fracture. (D)-(F) Hematoxylin and Eosin staining of histological slides

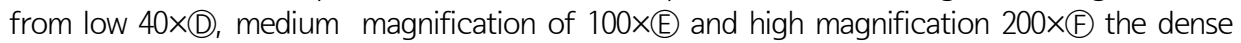
fibrous tissue (D)-(F) with organized collagen bundles populated by fibroblastic cells among haphazard layers of collagen $(\mathbb{F}$, black arrowheads) surround the neat layers of lamellae of bone which with the basophilic osteocytes $\left(\mathbb{F}_{1}\right.$, black arrow) suggestion of features of fracture and attempted bone healing.

nonunion of SAP tip fracture ${ }^{9)}$. Magnetic Resonance Imaging (MRI) demonstrated right $\mathrm{L} 1 / 2$ lateral recess stenosis with abutment of the traversing nerve root at the tip of superior articular process (SAP) (Fig. 2(A)-(D)). He was treated conservatively for several years with pain management and physiotherapy but did not improve. He underwent left Lumbar one Lumbar two Lumbar Endoscopic Unilateral Laminotomy For Bilateral Decompression (LE-ULBD) with contralateral approach to the right SAP with non-united bone and scar tissue resected. Intraoperatively we found the foraminal ligament was attached to the fragment and its displacement was causing compression on the traversing nerve root which was resected. The decompression and resection of the bony fragment was not expected to cause spinal instability hence no fusion nor is hardware instrumentation were deemed necessary. Post operatively his pain score improved from Visualized Analog Scale (VAS) 8 to VAS 0 . He returned to work in 2 weeks. His post-operative $C T$ and $M R I$ scan are as shown (Fig. 2(E)-(H).

\section{Patient 2}

A 74 years old retired lady, who complained of acute onset 
of lower back pain left buttock pain and left leg and calf pain for 1 month after a fall with background of lower back pain for years, she had vertebroplasty done one month ago which helped in her back pain but no improvement for her left leg buttock and left leg pain. Her Visual Analog Scale score on admission was 9 for her left leg pain. Physical examination was unremarkable with normal motor and sensory findings but limited by severe pain. XR done showed degenerative spondylosis in lumbar spine and $L 4$ compression fracture previously treated by vertebroplasty. CT showed vertebroplasty with compression fracture in L4 and left L5 SAP fleck of bone at the tip of SAP with a gap from the main part of $S A P^{9)}$. Corresponding MRI cut showed left $L 4 / 5$ Spinal Stenosis particularly at left $L 4 / 5$ lateral recess stenosis caused by the bony fragment (Fig. 4(A)-(D)). She attempted conservative treatment with no improvement and was unable to walk. She underwent LE-ULBD of L4/5 with contralateral approach to the left L5 SAP fragment resection (Fig. 4(A)-C). Her resected fragment was sent for histological evaluation, which showed dense fibrous tissue with organized collagen bundles populated by fibroblastic cells (Fig. 4(D)-(F). Her post-operative CT and MRI in the corresponding cut showed decompressed L4/5 segment (Fig. 4(D)-(F). Her clinical symptoms improved to VAS 0 for both back and leg VAS on one year follow up.

In both cases, the same surgical technique was applied despite different lumbar levels. We described the technical note for case 2 with a video and narration (Video 1).

Technical Note of Endoscopic Stenotic Lumbar Decompression of Left L4/5 with resection of left L5 superior articular process tip fragment.

Preoperatively, we obtained anteroposterior lumbar spine $\mathrm{X}$-ray with flexion, extension view to assess stability of the lumbar spine. CT and MRI scan were done to assess levels of stenosis and bony architecture. In this case, we found out the cause of patient's pain was contributed by stenosis and tip of the left L5 SAP fragment. We performed Lumbar Endoscopic Unilateral Laminotomy For Bilateral Decompression (LE-ULBD) of Left L4/5 with resection of left L5 superior articular process fragment. Patient was positioned in prone position on Wilson Frame under epidural anesthesia and conscious sedation. Single dose of antibiotics was administered in preoperative period.

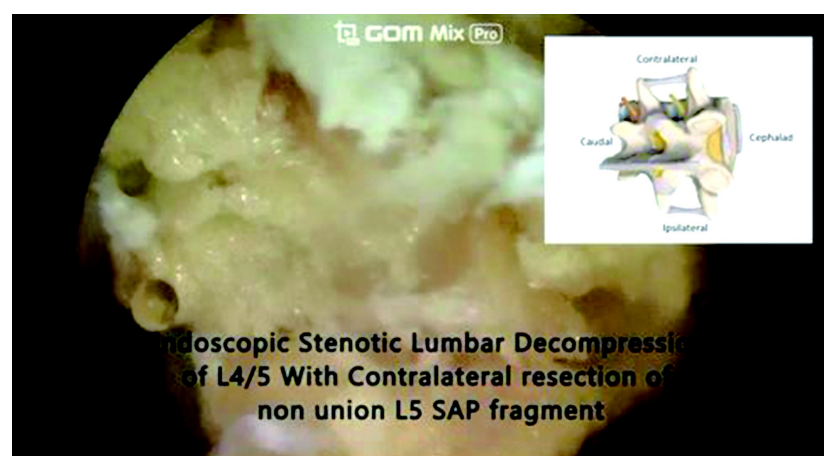

Video 1. SAP non union ESLD with Narration.
The entire procedure was performed under constant saline irrigation. We preferred to use arthropump with pressure set at $30-40 \mathrm{~mm}$ of $\mathrm{Hg}$ with $100 \%$ flow rate. We marked the $\mathrm{V}$ point on the right $L 4 / 5$ level which is the intersection of ipsilateral laminar window with medial inferior most point of the facet joint on fluoroscopy. $1 \mathrm{~cm}$ skin incision was made over the $\mathrm{V}$ point. A guidewire docked on the $\mathrm{V}$ point with gradual dilation and introduction of working cannula performed. ILESSYS-delta ${ }^{\mathrm{R}}$ (Joimax $\mathrm{GmbH}$, Germany) with outer diameter of $13.7 \mathrm{~mm}$ and open beveled tip endoscope which had a $15^{\circ}$ viewing angle, outer diameter of $10 \mathrm{~mm}$, working channel diameter of $6 \mathrm{~mm}$ and working length $125 \mathrm{~mm}$ was used. Bevel tip was docked over lateral bony structures with working cannula facing medially towards ligamentum flavum. We first worked on the $\mathrm{V}$ point, using endoscopic drills, Kerrison rongeurs, probe and forceps, we detached the ipsilateral attachment of ligamentum flavum to ipsilateral SAP. Drilling was continued on the ipsilateral cephalad lamina till ligamentum flavum attachment was loosened and we repeated the process for caudal attachment of ligamentum flavum. We detached the attachment of ipsilateral ligamentum flavum but kept it on the surface of dura to protect dura as a shield and worked on the contralateral ligamentum flavum. We then proceeded to detach the contralateral ligamentum flavum from its contralateral cephalad, SAP and caudal attachment with the use of endoscopic drill, Kerisson rongeurs and forceps. We further decompressed SAP for lateral recess decompression. We exposed the contralateral left L5 SAP tip fragment and separated it from its attachment to foraminal ligament and adhesion to the dura of traversing left $L 5$ nerve root. We teased it off gently with forcep and Kerisson Rongeurs. After removal of fragment, we inspected the dura for signs of decompression showing pulsatile dura under hydrostatic fluid and no deformation on any part of dura sac. We closed with a drain inserted in layers with sutures to skin.

Drain was removed on post-operative day 1 and patient was allowed to ambulate on same day post operatively.

\section{DISCUSSION}

Symptomatic superior articular process fracture (SAP) of lumbar spine is an uncommonly reported condition in the lite- rature ${ }^{1,7,14,19)}$.

Asymptomatic vertical cleft in superior articular process was reported to be of no clinical significance by Miki et al. ${ }^{12)}$. Ossicles of lumbar articular facet had been sporadically described in literature with a high prevalence in young athletic group compared to non-active group suggestive of as part of the spectrum of stress-induced changes in the posterior neural $\operatorname{arch}^{10)}$. While it was hard to decipher whether it was a development abnormality precipitated by exercise induced repetitive trauma or repetitive trauma leading to fracture nonunion as proposed by various authors, sports related injury is an association of these SAP tip fractures such as those reported in skier and skater ${ }^{7,16)}$. Their fracture characteristics were different in locations in L5 and Sacrum and in their symmetrical pattern as compared to 
our first case. The authors of both papers postulated the cause were repetitive stress injury to SAP. In our first case, the patient played golf regularly which might have contributed to his condition.

SAP fracture could have been a result of major trauma associated with injury of the subchondral bone plate, and tears of the articular capsule, including the ligamentum flavum of lumbar spine ${ }^{18,19)}$. These studies were done on post mortem cases with other serious injuries. Anatomical studies and concordant MRI studies described foraminal ligament attachment to the upper and anterior surface of the superior articular process of the lower corpus vertebra ${ }^{10,11)}$. In our second case, the SAP tip fracture might have resulted from trauma sustained after a fall in an osteoporotic vertebra with possible avulsion fracture of left L5 SAP from the foraminal ligament attachment. In elderly, ligament might be stronger in consistency than bone due to osteoporosis of the bone quality. During endoscopic procedure in this case, we could clearly see under endoscopic vision the attachment of the tip of the SAP fragment to the intravertebral foraminal ligament, the authors postulated that an avulsion fracture of SAP tip from the foraminal ligament is a possible mechanism which was not described as far as we know in the literature.

It is well established that facet joint is supplied by many sensory nerves and nociceptive nerve endings. It is a common site for pain management with facet joint block ${ }^{3,111}$. Nonunion of SAP can lead to pain in various mechanisms such as mechanical pain with movement secondary to attachment of foraminal ligament and ligamentum flavum ${ }^{2}$, inflammation and irritation secondary to repetitive injuries and non-healing of the nonunion site $e^{8,17)}$.

Treatment options varied in the literature. Numoto et al. performed open decompressive resection of the affected fragment without fusion ${ }^{14)}$ while Kojima and Renato et al. performed fusion procedure for their patients, however in their cases the superior articular facet defects were bilateral ${ }^{1,7)}$. Nakamura et al. described inferior articular facet stressed fracture which 6 out of 7 cases are treated with conservative treatment ${ }^{13)}$. To the best of our knowledge, this is the first report to describe lumbar superior articular facet fracture causing intractable back and right gluteal pain treated with endoscopic decompression and resection of SAP nonunion. Uniportal LE-ULBD with contralateral approach allows clear direct visualization of the fragment and its attachment to the foraminal ligament ${ }^{6}$, ensuring optimal resection of fragment to relieve symptoms while preserving facet joint for stability. With the evolution of endoscopic spine surgery and better visualization equipment and instruments for resection, it can be considered as a viable options among the options available in literature.

\section{CONCLUSION}

Symptomatic fracture of superior articular process can cause significant morbidity to patients. It can be missed if care is not taken to look carefully for small fleck of bone on the supe- rior articular process of lumbar spine. Uniportal endoscopic contralateral approach decompression is a viable treatment option with conservation of the facet joint function and good visualized retrieval of non-united fragment with selective decompressive resection.

\section{ACKNOWLEDGMENTS}

We would like to acknowledge scientific team members Ms. Jae Eun Park, Mr. Kyeong-rae Kim, Ms. Elin Lee and Mr. Sang Hyuck Yoon for providing assistance in acquiring full text articles and managing digital works.

Compliance with Ethical Standards Funding: No funds were received in support of this work.

No benefits in any form have been or will be received from a commercial party related directly or indirectly to the subject of this manuscript.

Conflict of Interest: all co-authors have no conflict of interest.

Ethical Approval: All procedures performed in studies involving human participants were in accordance with the ethical standards of the Nanoori Hospital's Ethics Committee and the national research committee and with the 1964 Helsinki declaration and its later amendments or comparable ethical standards.

\section{REFERENCES}

1. Bosita RV, Hong T, Ohnmeiss DD: Avascular necrosis of lumbar facet joints associated with bilateral facet fractures. Spine J 9: e1-4, 2009

2. Caglar YY, Dolgun H, Ugur HC, Kahilogullari G, Tekdemir I, Elhan A: A ligament in the lumbar foramina: inverted Y ligament: an anatomic report. Spine (Phila Pa 1976) 29:1504-1507, 2004

3. DuToit N, Mitchell B, Joseph J, Barnard A: Movement and muscle activation patterns following medial branch blocks for facet joint pain, and sacroiliac injection for sacroiliac joint pain. Journal of Science and Medicine in Sport 19:e46-e46, 2016

4. Grazina R, Andrade R, Santos FL, Marinhas J, Pereira R, Bastos $\mathrm{R}$, et al: Return to play after conservative and surgical treatment in athletes with spondylolysis: A systematic review. Physical Therapy in Sport 37:34-43, 2019

5. Haher TR, O'Brien M, Dryer JW, Nucci R, Zipnick R, Leone DJ: The role of the lumbar facet joints in spinal stability. Identification of alternative paths of loading. Spine 19:2667, 1994

6. Kim H-SMDP, Paudel BMDMS, Jang J-SMDP, Oh S-HMDP, Lee SBE, Park JEBS, et al: Percutaneous full endoscopic bilateral lumbar decompression of spinal stenosis through uniportal-contralateral approach: Techniques and preliminary results. World Neurosurgery 103:201-209, 2017

7. Kojima K, Asamoto S: Bilateral fracture of the superior articular process of S1 - An unusual fracture seen in a speed skater. $\mathrm{Br}$ J Neurosurg 31:273-274, 2017

8. Kras JV, Dong L, Winkelstein BA: Increased interleukin-1a and prostaglandin E2 expression in the spinal cord at 1 day after 
painful facet joint injury: Evidence of early spinal inflammation. Spine 39:207-212, 2014

9. Kuhlman JE, Fishman EK, Magid D, Scott WW, Jr., Brooker AF, Siegelman SS: Fracture nonunion: CT assessment with multiplanar reconstruction. Radiology 167:483-488, 1988

10. Kumar DS, Fotiadou A, Lalam R, Ginder LM, Eisenstein SM, Tins BJ, et al: Ossicles of lumbar articular facets: normal variant or spondylolytic variant? Skeletal Radiology 41:1559-1566, 2012

11. Manchikanti L, Singh V, Falco FJE, Cash KA, Pampati V: Lumbar facet joint nerve blocks in managing chronic facet joint pain: one-year follow-up of a randomized, double-blind controlled trial: Clinical Trial NCT00355914. Pain Physician 11:121, 2008

12. Miki T, Oka M, Hama H, Shima M, Hirofuji E, Tanaka S: Vertical cleft through the superior articular process of the lumbar spine: fracture or anomaly? Case report. J Neurosurg 53:406407, 1980

13. Nakamura T, Ikeda T, Takagi K: Defect in the articular process of the lumbar facet. Journal of Spinal Disorders \& Techniques 15:550-554, 2002

14. Numoto RT, Takeda M, Tani S, Abe T: Fractures of the lumbar and sacral superior articular processes: report of two cases. Neu- rosurgery 56:193, 2005

15. O'Leary SA, Paschos NK, Link JM, Klineberg EO, Hu JC, Athanasiou KA: Facet joints of the spine: Structure-function relationships, problems and treatments, and the potential for regeneration. Annual Review of Biomedical Engineering 20:145-170, 2018

16. Omar MM, Levinson EM: An unusual fracture of the vertebral articular process in a skier. J Trauma 19:212-213, 1979

17. Tachihara H, Kikuchi S-i, Konno S-i, Sekiguchi M: Does facet joint inflammation induce radiculopathy?: an investigation using a rat model of lumbar facet joint inflammation. Spine 32:406412, 2007

18. Taylor JR, Twomey LT, Corker M: Bone and soft tissue injuries in post-mortem lumbar spines. Paraplegia 28:119-129, 1990

19. Twomey LT, Taylor JR, Taylor MM: Unsuspected damage to lumbar zygapophyseal (facet) joints after motor-vehicle accidents. Med J Aust 151:210-212, 215-217, 1989

20. Wiersbicki D, Völker A, Heyde C-E, Steinke H: Ligamental compartments and their relation to the passing spinal nerves are detectable with MRI inside the lumbar neural foramina. European Spine Journal 28:1811-1820, 2019 